\title{
EL PROFESOR NOVEL ANTE LA INSERCIÓN PROFESIONAL A LA ENSENAANZA EN EDUCACIÓN MEDIA SUPERIOR: MODELO DE CHOQUE DIVERGENTE
}

\author{
Erick Zorobabel Vargas Castro* \\ Universidad Pedagógica del Estado de Sinaloa
}

\section{RESUMEN}

En este artículo se exponen los resultados de una investigación hecha con profesores principiantes de Educación Media Superior (EMS) en la ciudad de Mazatlán, Sinaloa, México. Se analiza la inserción experimentada por sujetos egresados de distintas áreas del conocimiento quienes, careciendo de una formación ad hoc y alejados de su "hábitat natural» profesional, se ven condicionados por su perfil de egreso, la percepción de los ámbitos más ambiguos de la práctica y una cultura institucional que omite proveerles «acompańamiento» al dar por sentado que superarán los problemas «naturales» de este periodo conforme descubran su cotidianidad. Las sensibles diferencias registradas permitieron diseńar un modelo explicativo con enlaces divergentes sobre el efecto «choque con la realidad» (Veenman, 1984) según la articulación de diversos factores (relación, subordinación, cooperación) para percibirlo con mayor o menor intensidad.

PAlabras Clave: formación docente, inserción profesional, profesor novel, choque con la realidad.

\section{THE BEGINNER TEACHER BEFORE THE PROFESSIONAL INSERTION TO TEACHING} IN HIGHER SECONDARY EDUCATION: DIVERGENT SHOCK MODEL

\section{Abstract}

In this article we expose the results of an investigation made with beginning teachers of Higher Secondary Education in the city of Mazatlán, Sinaloa, Mexico. The insertion experienced by subjects graduated from different areas of knowledge is analyzed who, lacking an ad hoc training and far from their professional "natural habitat", are conditioned by their graduation profile, the perception of the most ambiguous areas of practice and an institutional culture that fails to provide "accompaniment" by assuming that they will overcome the "natural" problems of this period as they discover their daily life. The sensitive differences registered, allowed to design an explanatory model with divergent links on the effect «reality shock» (Veenman, 1984) according to the articulation of various factors (relationship, subordination, cooperation) to perceive it with greater or lesser intensity. KEYWORDS: teacher training, professional insertion, beginner teacher, reality shock. 


\section{INTRODUCCIÓN}

Las abstracciones que apuntalan esta propuesta teórica, iniciadas en el 2006, se enfocaron en la Etapa de Inserción a la Enseñanza (EIE) por ser un campo problemático poco estudiado en contexto. Si bien a la fecha (2018), el Sistema Educativo Mexicano (SEM) incluyó en la última Reforma Educativa (2013) la figura del «Tutor» para profesores que ingresan por primera vez al Servicio Profesional Docente en Educación Básica (EB) y EMS, su implementación no ha sido del todo integral, regulada o progresiva, visto que existe una mayor atención hacia la $\mathrm{EB}$, tuvo escasa capacitación previa, no cuenta con personal suficiente que la ejerza, tiene un limitado presupuesto para otorgar estímulos, se encuentra alejada de los ciclos de la formación docente y sigue distanciado el binomio teoría-práctica entre Instituciones Formadoras y Actualizadoras de Docentes (IFAD) y Centros Escolares; a pesar de su trascendencia por significar un periodo puente entre la Formación Inicial (FI) y el Desarrollo Profesional (DP).

Por lo que respecta al periodo de inserción en la EMS, aun cuando este primer momento de socialización profesional comparte rasgos con los niveles educativos precedentes, posee algunos exclusivos que definen el tránsito de quienes se integran por primera vez a su planta docente, en este caso, sujetos formados en distintas áreas del conocimiento (físico-matemáticas, ciencias de la salud, ciencias sociales), sin haber confrontado un contexto donde convergen alumnos, rutinas, tecnicismos, carga administrativa y la responsabilidad del proceso enseñanza-aprendizaje; asimismo, "sumergirse» en esta cultura escolar significa adentrarse en un escenario que conocieron como estudiantes pero desconocen como profesores (Collins \& Winnip, 2002), lo cual obliga a constituir una identidad profesional distinta a su formación de origen, en ocasiones ejerciendo ambas paralelamente.

Dicho escenario «inexplorado» implica confrontar múltiples problemas emergentes cuya naturaleza da forma al efecto "choque con la realidad» (constructo teórico acuñado durante la década de los 80 del siglo xx por Simon Veenman) característico de los primeros años de enseñanza profesional, el cual involucra, en gran medida, un «abrazo inaugural» bajo las peores condiciones laborales padeciendo presiones de diversa índole, inseguridad, necesidad de aprender y sobrevivir mientras se ven trastocados ámbitos físicos, emocionales, psicológicos, cognitivos, económicos o familiares, siendo reflejo de una etapa contradictoriamente relevante y desestimada por los centros escolares y las IFAD al no haberse aprovechado la experiencia del profesor novel para el diseño de programas oficiales.

*E-mail: erick.vargas@upes.edu.mx. Es maestro en Educación por la Universidad Pedagógica Nacional, candidato a doctor en Desarrollo Educativo por la Universidad Pedagógica del Estado de Sinaloa (UPES), ha presentado ponencias en congresos de México, Argentina, Chile y Brasil, autor de diversos artículos de revistas y capítulos de libros; su línea de investigación se enfoca en la Inserción Profesional a la Enseñanza, el Profesor Novel, los Saberes Docentes y el proceso de Aprender a Enseñar. Es integrante del Comité Ejecutivo del Centro de Estudios de Política Educativa y Gestión Universitaria de la Universidad Nacional de Cuyo. Fue director de la Unidad Académica de UPES Mazatlán, en la que actualmente se desempeña como profesor investigador. 
Aunque el constructo «choque con la realidad» ha tenido una mayor aplicación en la EB (donde sus profesores parten de una FI), al transferirlo hacia la EMS posibilitó advertir algunas salvedades, es decir, mientras que en la EB se contrasta el valor de los conocimientos obtenidos como alumno durante la FI y las experiencias que provee la práctica profesional una vez asumida la titularidad de un grupo, en la EMS los profesores ingresan a una "realidad escolar» apoyados en "modelos docentes» observados en su trayectoria escolar previa (incluida la educación superior) desde los que interiorizan conocimientos a manera de "equipo de supervivencia» (Gilroy, 1993) reproducibles, así como el sustento de una idea personal de la práctica, a partir de dichos modelos, con la que efectúan un autocontraste entre idealización del ejercicio profesional versus descubrir una ríspida «realidad» en contexto.

En este sentido, la EMS se encuentra diversificada en tantas y tan variadas instituciones dentro del contexto mexicano, cada una siguiendo las directrices específicas del subsistema al que pertenece, que la integración de los nuevos profesores apegada a usos y costumbres anquilosados sin considerar sus necesidades formativas, la adaptación al centro escolar, deficiencias para interactuar con los alumnos, asegurar el aprendizaje, dosificar contenidos, sobrecarga burocrática, etc., motivaron investigar esta visión simplificada que subvalora la inserción como objeto de estudio, sin distingo del profesor novel con formación docente o sin ella, según las características que expusiera confrontar la "realidad escolar» y el «choque» generado al insertarse en la enseñanza profesional.

\section{REFERENTE TEÓRICO}

Posicionados en el paradigma del Pensamiento del Profesor (Clark \& Yinger, 1980; Marcelo, 1987; Imbernón, 2007), la investigación recuperó sus planteamientos acerca de la concordancia que tienen conocimiento práctico, constructos personales, saberes, nociones previas y teorías implícitas en el ejercicio de la profesión docente; su influencia sobre el trabajo realizado en el aula, la toma de decisiones o la resolución de problemas en contexto; dado que la complejidad donde vive e interactúa a diario el profesor implica que sus procesos de pensamientos no se produzcan en vacío (Clark \& Yinger, 1979), sino intercalados entre un contexto psicológico (conocimientos, teorías implícitas, valores, creencias) y uno ecológico (recursos, circunstancias externas, limitaciones administrativas).

Esta progresión de pensamientos se desarrolla en el ejercicio de su actividad diaria, es decir, la enseñanza, considerada en este paradigma como una actividad altamente cognitiva que requiere dominar un amplio nivel de competencia si se tiene en cuenta el sinfín de decisiones que toma dentro de un ambiente tan dinámico y complejo (Berliner, 1984) como es el aula, aunado a la heterogeneidad de su grupo, el nivel educativo, su nivel de experiencia, el contexto, la cultura del centro escolar; de tal suerte que permite asumirla como una profesión al traslapar diversas fuentes de información extraídas de un conocimiento fundamentado en la experiencia, los estudiantes, las limitaciones que vive en el día a día, así como las previsiones que hace de futuras situaciones y condensa en decisiones pedagógicas (Hunter, 1984). 
Acorde con lo anterior, todas las particularidades de su trayecto vivencial, escolar y profesional dan cuenta del permanente proceso formativo que recorre el profesor mientras edifica una identidad docente, sin distingo del nivel educativo donde labore, el cual integra un largo proceso de aprendizaje secuenciado entre diferentes etapas que va modulándose ante la percepción de conflictos con distintos niveles de afectación según el tiempo y espacio en que se generen; en este sentido, se recuperó la clasificación hecha por Marcelo (1992) de la formación docente articulada en cuatro etapas, cada una con problemas y preocupaciones relacionados con el momento de formación, el estatus laboral, su lugar de ejercicio, la adquisición de experiencia; entre otros, dividida en a) Preformativa, b) Formación Inicial, c) Iniciación o Inserción a la Enseñanza y d) Desarrollo Profesional.

En lo referente a la EIE, es un periodo que ha recibido una ambigua atención desde finales del siglo Xx, una vez advertida su importancia formativa por ser el eslabón que unía la FI con el DP (Marcelo, 1999). Si bien representa el contacto inaugural de los profesores con la "realidad escolar», ejerciendo la enseńanza profesional desde el súbito tránsito de estudiante en formación a profesor autónomo (Vonk, 1996), recorrerla no significa dar un "salto al vacío» sino un momento clave para el entendimiento de la profesión; por ello, reconocerla como eslabón formativo constituye una de sus mayores características. No obstante, puede prescindir de esta función cuando no se cursa una FI y se asume de lleno el papel reservado a los profesores (Marcelo, 2007), en este caso, convirtiéndose en el punto de partida del futuro profesional de la enseñanza que se edificará.

Por otra parte, los primeros años de enseñanza definen una etapa tensa con aprendizajes intensivos en contextos generalmente desconocidos (Marcelo, 1999), se caracteriza desde el primer año por el desarrollo del ejercicio de la enseńanza mediante un proceso de ensayo-error, un marcado sentimiento de supervivencia y el predominio del valor hacia lo práctico (Veenman, 1984); además, estos años constituyen el «ritual» que transmite la cultura docente al profesor novel e integra la cultura escolar en su personalidad para adaptarse al entorno social donde efectúa sus actividades mientras «enseña» pero también «aprende», en todo caso, bajo el modelo «nada o húndete» (Marcelo, 1999), de tal suerte que llega constituir uno de los lapsos más «ríspidos» del proceso amplio y flexible que representa la formación docente.

Este último rasgo es relevante para comprender el efecto del «choque con la realidad» (Veenman, 1984), propio de la EIE, que en términos generales indica el colapso de los ideales misioneros adquiridos durante la FI ante la cruda y áspera realidad de la vida escolar cuando se asumen por completo las responsabilidades como profesor frente a grupo, no siendo fortuito que llegue a convertirse en una experiencia tan desastrosa y traumática que demanda transformar las creencias e ideas sobre la enseñanza hacia otras para justificar la propia práctica (Vonk, 1983), en términos generales, situando al profesor novel en la premura de resolver de manera autónoma una vorágine de situaciones emergentes solo con los «recursos» que haya adquirido en las etapas de formación previas.

Así pues, iniciar la enseñanza profesional conlleva para el profesor novel la convergencia del aprendizaje interiorizado a lo largo de la vida (Day, 2005) mientras cumple dos tareas, enseñar y aprender a enseñar, acompañadas con la bús- 
queda de su propia identidad tanto personal como profesional (Esteve, 1997); no obstante, como algunos pormenores de la enseñanza solo se aprenden en la práctica (Feinman-Nemser, 2001) necesita desarrollar con rapidez conocimientos profesionales y conservar un equilibrio emocional durante estos primeros ańos, que son de supervivencia, descubrimiento, adaptación, aprendizaje y transición (Marcelo, 2008), a fin de alcanzar un óptimo desarrollo profesional apuntalado sobre el axioma del continuum interactivo (Bullough, 2000; Huberman, Thompson \& Weiland, 2000).

Por ello, el factor cognitivo-emocional se convierte en uno que redefine el ejercicio del profesor novel cuando confronta por primera vez la «realidad escolar» al percibir distintos niveles de placer o malestar, esto es, Disonancia Cognitiva (Festinguer, 1957), dado que la interacción con un contexto ajeno al marco de referencias propio, una creencia u opinión de sí mismo, promoverá tomar decisiones «incoherentes» apremiado por la incertidumbre de su efectividad en la enseñanza si producen resultados adversos, no siendo fortuito que reducir el nivel de ansiedad resentido en dichos lapsos sea una necesidad permanente cuando trata de procesar información e ideas que entran en conflicto; por ello, la dificultad para encontrar las razones de por qué esta urdimbre no encaja entre sí (disonancia) obtendrá una respuesta automática hasta lograr que se empalmen de alguna manera (reducción de la disonancia).

\section{OBJETO DE ESTUDIO}

Como se precisó, fueron analizadas las particularidades que adquiere la inserción a la enseñanza del profesor novel al comienzo de su ejercicio en la EMS, bajo la premisa de que concurre un «impacto» desigual si se tiene en cuenta que son distintos profesionistas o profesionales de la educación, sin formación inicial ad hoc, quienes confrontan las necesidades cognitivas, emocionales, psicológicas, conductuales, etc., de alumnos entre los 15 y 18 años de edad, así como el hecho de que el escenario «natural» donde ejercen (una obra en construcción, un despacho o consultorio) difiere de la cultura e identidad propia de la enseńanza y los centros escolares; siendo referentes sustantivos para percibir el «choque con la realidad» conforme emergieran las nociones personales de la «enseñanza» y un contexto en apariencia hostil que daría su abrazo inaugural.

En este sentido, se documentaron los problemas y preocupaciones de profesores noveles durante sus tres primeros ańos de enseñanza profesional, incorporados en la diversidad de subsistemas que integran la EMS según fueron «descubriendo» las múltiples situaciones conflictivas que formaban parte de sus actividades intra- e interáulicas, a fin de identificar las recurrencias sobre vacíos formativos, sobrecarga docente, apoyo institucional, control de la disciplina, dominio de contenidos, planeación de las actividades, organización del trabajo de clase, insuficiencia de materiales, el contacto vis a vis con alumnos, como fundamento de una escala jerárquica con la que se contrastaran las diferencias o similitudes resultantes entre dichos ámbitos de la práctica más problemáticos. 
Para acotar el problema de investigación, la selección de profesores noveles incluyó dos tipos de perfiles según su formación de origen, esto es, uno integrado por profesionistas universitarios egresados de distintas áreas de conocimiento, sin experiencia docente previa, y otro con profesionales de la enseńanza egresados de IFAD pertenecientes al estado de Sinaloa, ambos laborando por primera vez como profesores frente a grupo en bachilleratos del Municipio de Mazatlán con un máximo de tres ańos de ejercicio profesional docente, en concreto, para responder la pregunta de investigación ¿qué ámbitos de influencia afecta el «choque con la realidad» escolar en los profesores noveles que tienen una formación docente $\mathrm{y}$ en los que no la tienen?

\section{METODOLOGÍA}

Se adoptó el enfoque mixto para el manejo de los datos en la idea del continuum interactivo (Newman \& Bens, 1998; Tashakkori y Teddlie, 1998), utilizando el enfoque Biográfico-Narrativo (Connelly \& Clandinin, 1995; Minichiello, Aroni, Timewell \& Alexander, 1990; Bolívar y otros, 1998) en su modalidad life review o revisión de vida (Booth, 1996), con el que se obtuvieron relatos de primera mano sobre sus experiencias docentes en las primeras semanas de trabajo, además de una entrevista estructurada (Aguirre, 1995) para establecer el rapport con los participantes y desarrollar dos áreas de exploración. El sistema de categorías utilizado fue la Parrilla de Veenman (Eirín, Montero \& García, 2008).

A partir de las narrativas elaboradas por los participantes, se efectuó una criba con la información para identificar ámbitos problemáticos relacionados con Planeación, Contenidos, Trabajo de Clase, Estrategias de Enseñanza, Materiales/Recursos Didácticos, Motivación, Disciplina, Nivel de Aprendizaje, Apoyo Institucional y Relaciones Interpersonales; desde los que se diseñó el guion de una entrevista focalizada para profundizar en sus particularidades, organizando la nueva información en Categorías Básicas que agruparon problemas de a) Interacción Alumno-Profesor, b) Disciplina en el Aula, c) Formación, d) Carácter Institucional, e) Extraclase y f) Carácter Relacional.

\section{PARTICIPANTES}

La selección de participantes incluyó 40 profesores ( 15 hombres y 25 mujeres) laborando frente a grupo en instituciones de los subsistemas federal (Bachillerato Tecnológico e Industrial), estatal (Bachillerato del estado de Sinaloa) autónomo (Preparatoria Autónoma Universitaria) y Privado (Bachillerato General). Los profesores sujetos de investigación se organizaron en cuatro grupos según la coincidencia entre Áreas de Conocimiento (UNAM, 2009) conforme lo siguiente: 1) Ciencias Biológicas y de la Salud (CBS), 2) Ciencias Físico-Matemáticas e Ingenierías (CFMI), 3) Ciencias Sociales (CS) y 4) Educación (ED). Cada grupo fue integrado por diez profesores cuyo tiempo de ejercicio abarcara desde nuevo 
ingreso hasta los tres años como máximo. Si bien el grupo cuatro no representaba un área de conocimiento específica por pertenecer a las Ciencia Sociales, se decidió separarla para concentrar las licenciaturas en Educación, Pedagogía y Ciencias de la Educación.

\section{CONTRASTE INTERGRUPAL}

El resultado del análisis entre áreas de conocimiento derivó en constituir una tabla de doble entrada por cada Categoría Básica que incluye sus Categorías Subordinadas en orden jerárquico al significarles un mayor malestar para su práctica docente durante los primeros contactos con la «realidad escolar», de modo que se presentan a continuación las semejanzas y similitudes de los ámbitos que resintieron de mayor a menor dificultad en el siguiente orden:

\begin{tabular}{ccccc}
\hline \multicolumn{5}{c}{ TABLA 1. PROBLEMAS DE FORMACIÓN (PF) } \\
\hline \multicolumn{7}{c}{ CBS } & CFMI & CS & ED \\
\hline 1 & Conoc. del Cont. & Org. Trabajo de Clase & Conoc. del Cont. & Conoc. del Cont. \\
\hline 2 & Org. Trab. de Clase & Conoc. del Cont. & Org. Trab. de Clase & Org. Trab. de Clase \\
\hline 3 & Planif. Ens.-Apr. & Ut. Ef. Metds. Ens. & Planif. Ens.-Apr. & Ut. Ef. Metds. Ens. \\
\hline 4 & Ut. Ef. Metds. Ens. & Ut. Ef. LyG & Ut. Ef. Metds. Ens. & Ut. Ef. LyG \\
\hline 5 & Ut. Ef. LyG & Planif. Ens.-Apr. & Ut. Ef. LyG & Planif. Ens.-Apr. \\
\hline
\end{tabular}

Contraste por categoría subordinada.

Fuente: elaboración propia.

Las cuatro áreas sostuvieron los PF como la categoría básica con el mayor porcentaje documentado, convirtiéndose en fuente de sus mayores preocupaciones y desavenencias al iniciar el ejercicio de la enseñanza, lo cual refleja una tendencia en EMS hacia la formación insuficiente y los diversos factores que la rodean para valorarlos como aspectos que necesitan ser cubiertos o dominados con una mayor aptitud en el menor tiempo posible; ahora bien, con excepción de CFMI, las tres áreas restantes (CBS, CS, ED) afirmaron que su dominio del contenido curricular, elemento primordial que apuntalaba la enseñanza, era insuficiente aunado a desconocer cómo se organizaba el trabajo de clase.

Por otro lado, la actividad de planeación para las CBS resintió un desconocimiento generalizado a diferencia de ED, lo cual sería hasta cierto punto entendible por tratarse de profesionales de la educación a pesar de no tener experiencia en la EMS; sin embargo, también fueron valoradas por las CFMI con menor jerarquía por considerar que el conocimiento técnico propio era suficiente para solventar este requisito previo; asimismo, las cuatro áreas percibieron que era valuada su práctica según utilizaban con eficiencia las estrategias de enseñanza, libros o guías docentes para aclarar dudas en cada clase y presentarse ante sus alumnos como un docente bien preparado. 


\begin{tabular}{|c|c|c|c|c|}
\hline & CBS & CFMI & CS & ED \\
\hline 1 & Disciplina Clase & Disciplina Clase & Disciplina Clase & Disciplina Clase \\
\hline 2 & Manejo Est. Probl. & Manejo Est. Probl. & Manejo Est. Probl. & Manejo Est. Probl. \\
\hline 3 & Elev. No. Alumnos & Elev. No. Alumnos & Elev. No. Alumnos & \\
\hline
\end{tabular}

De la misma manera, se documentaron las PDA en segundo lugar de jerarquía para las cuatro áreas de conocimiento, en tanto descubrieron un ambiente disonante y conflictivo en el aula desde el primer momento de comenzar a dar clases, que por momentos era insoportable, de ahí que la indisciplina de clase, el manejo de estudiantes problemáticos y el elevado número de alumnos significaron agravantes conforme avanzaba el ciclo escolar hasta sufrir por momentos una incapacidad frustrante al no poder controlarlo. Además, en la mayoría de las narrativas estuvieron presentes los relatos sobre alumnos definidos como "líderes de la indisciplina», quienes una vez identificados eran sujetos a múltiples medidas para vigilarlos o castigarlos y evitar que se «disparara» el desorden durante las horas de clase, al tiempo que trataban de demostrar su autoridad como profesores.

\begin{tabular}{|c|c|c|c|c|}
\hline & CBS & CFMI & CS & ED \\
\hline 1 & Motiv. Estd. & Trab. Dif. Ind. Alu. & Motiv. Estd. & Motiv. Estd. \\
\hline 2 & Trab. Dif. Ind. Alu. & Motiv. Estd. & Trab. Dif. Ind. Al. & Trab. Dif. Ind. Alu. \\
\hline 3 & Valorar Trab. Est. & Deter. Nivel Apr. & Deter. Nivel Apr. & Valorar Trab. Est. \\
\hline 4 & Deter. Nivel Apr. & Valorar Trab. Est. & Valorar Trab. Est. & Deter. Nivel Apr. \\
\hline
\end{tabular}

Contraste por categoría subordinada.

Fuente: elaboración propia.

La siguiente categoría básica identificada de mayor intranquilidad no presentó la misma jerarquización para todas las áreas de conocimiento. En específico las CBS(3), CS(4) y ED(4) resintieron complicaciones con las PIAP durante las primeras semanas de práctica al no lograr que los alumnos se motivaran hacia su(s) materia(s), ni sabían cómo trabajar con sus diferencias y en menor medida evaluarlos con la mayor objetividad posible o valorar el trabajo realizado por cada uno de ellos; en contraparte, las CFMI(3) jerarquizaron con mayor dificultad enseñarle a un grupo heterogéneo de alumnos, seguido de motivarlos y determinar el nivel de aprendizaje como las categorías subordinadas con menores recurrencias bajo diversos niveles de preocupación.

\begin{tabular}{lcccc}
\hline \multicolumn{4}{c}{ TABLA 4. PROBLEMAS EXTRACLASE (PEC) } \\
\hline \multicolumn{2}{c}{ CBS } & CFMI & CS & ED \\
\hline 1 & Inad. Ases. y Apo. & Inad. Ases. y Apo. & Inad. Ases. y Apo. & Inad. Ases. y Apo. \\
\hline 2 & Insuf. Tpo. PCDoc & Insuf. Tpo. PCDoc & Sobrecarga Doc. & Insuf. Tpo. PCDoc \\
\hline 3 & Sobrecarga Doc. & Sobrecarga Doc. & Insuf. Tpo. PCDoc & Sobrecarga Doc. \\
\hline $\begin{array}{l}\text { Contraste por categoría subordinada. } \\
\text { Fuente: elaboración propia. }\end{array}$ & & & \\
\end{tabular}


En cuarto lugar, encontramos una marcada diferencia entre áreas sobre la categoría básica que les generó un número considerable de preocupaciones. Para el área de CBS las PEC estuvieron ubicadas en cuarto nivel de jerarquía a partir del inadecuado asesoramiento y apoyo obtenido por el centro escolar, una falta palpable de soporte emocional o laboral y algunos de sus compañeros de trabajo. Complementando este ámbito, algunos casos evidenciaron una dificultad práctica por la sobrecarga docente que derivaba en falta de tiempo para su planeación y preparación. Las áreas de CFMI, CS y ED(5) determinaron a las PEC en quinto lugar al percibir dificultad en su diario desempeño, señalando el inadecuado asesoramiento recibido desde su llegada al centro escolar, un insuficiente tiempo para planear la carga docente y haber sido sobrecargados de grupos.

\begin{tabular}{|c|c|c|c|c|}
\hline \multicolumn{5}{|c|}{ TABLA 5. PROBLEMAS DE CARÁCTER INSTITUCIONAL (PCI) } \\
\hline & CBS & CFMI & CS & $\mathrm{ED}$ \\
\hline 1 & Conoc. NyC Esc. & Conoc. $\mathrm{NyC}$ Esc. & Conoc. NyC Esc. & Conoc. $\mathrm{NyC}$ Esc. \\
\hline 2 & & Insuf. Materiales & Inad. Equip. Escolar & Sobrec-Inad-Insuf. \\
\hline 3 & & Sobrec. Trab. Buroc. & & \\
\hline 4 & & Inad. Equip. Escolar & & \\
\hline
\end{tabular}

Contraste por categoría subordinada.

Fuente: elaboración propia.

Por otro lado, los ámbitos generadores de incomodidades relacionados con el centro escolar tuvieron una jerarquización más diversa, esto es, las CS(3) y ED(3) relacionaron los PCI con las particularidades que envolvía el trabajo docente en la EMS al haber generado diversos niveles de ansiedad por desconocer las normas y costumbres formales e informales de cada centro escolar, no estar al tanto de su organización, tener que preguntar a los alumnos por la ubicación de sus instalaciones, no contar con un adecuado equipamiento escolar, así como la insuficiencia de materiales necesarios para el trabajo diario o la sobrecarga del trabajo burocrático que formaba parte de sus responsabilidades.

Respecto de las CFMI(4), ignoraban las diversas rutinas que estaban obligados a cumplir como la firma de asistencia diaria, los reportes de inasistencias a los prefectos o la autorización para utilizar equipo electrónico, siendo motivos que dificultaron su práctica sobremanera; además, mencionaron el ámbito sobrecarga docente como un problema constante por la correspondiente falta de tiempo para su organización; empero, a diferencia de las demás áreas se documentó la recurrencia de todas las categorías subordinadas que lo integraban. Ahora bien, las CBS(5) solo pronunciaron problemas con la normatividad que regía el diario acontecer sin que alguna autoridad se la diera a conocer; por ello, las PCI fueron consideradas motivos de dificultad relacionados con actividades rutinarias al momento en que comenzaron a trabajar. 


\begin{tabular}{|c|c|c|c|c|}
\hline \multicolumn{5}{|c|}{ TABLA 6. PROBLEMAS DE CARÁCTER RELACIONAL (PCR) } \\
\hline & CBS & CFMI & CS & ED \\
\hline 1 & Relac. Colegas & Relac. Colegas & Relac. Colegas & Relac. Colegas \\
\hline 2 & Relac. Director & Relac. Director & Relac. Director & Relac. Director \\
\hline
\end{tabular}

Al final de la escala jerárquica ubicamos en el sexto lugar de importancia a las PCR para todas las áreas con un mínimo de recurrencias a comparación de las demás categorías subordinadas, tanto en narrativas como en las respuestas de sus entrevistas, si bien esto significa que no fue un ámbito que generara mayores dificultades se considera un reflejo indeleble de los usos y costumbres que enmarca el proceso de inserción con interacciones entre compañeros de trabajo o directivos bajo un ambiente de trabajo donde experimentan no solo un nulo apoyo sino además aislamiento, críticas, menosprecio, etcétera.

Para las CBS existieron altas expectativas de entablar un primer acercamiento con sus compañeros de trabajo en lo personal o profesional si compartían algunos consejos sobre temas curriculares, metodológicos, disciplinarios, planificación u organización de actividades y facilitara su integración; empero, no obtuvieron algún apoyo emocional o práctico para sobrellevar la disciplina de su(s) grupo(s) cuando sentían que los rebasaban y por el contario solo recibieron sendas advertencias acerca de sostener trato con colegas identificados como problemáticos o malos ejemplos.

Las CFMI afirmaron no haber tenido en principio una buena relación con otros profesores quienes emitían diversas críticas enfocadas específicamente hacia la eficacia de su trabajo por ser noveles e inclusive esto parecía promover una «competencia» entre profesores que se encontraban en igualdad de ingreso para demostrar su capacidad. En algunos casos dichas críticas les motivó asesorarse con algún profesor conocido o amigo de su familia a quienes les pedían consejos sobre cómo enseñar, organizar los temas curriculares, dosificar las clases y controlar la disciplina de grupo, siendo asumida como una «tablita de salvación» esta ayuda incondicional.

Las relaciones entre las CS y los colegas representaron un motivo de malestar significativo durante los primeros días de trabajo docente ante las constantes expresiones de menosprecio hacia su desconocimiento de la enseńanza, manifestado por profesores con más años de servicio, de tal suerte que una manifiesta juventud relacionada con inexperiencia suponían elementos de crítica al considerarlos incapaces de realizar un trabajo decoroso; paradójicamente, ese entorno de hostilidad emplazó demostrar lo contrario, motivándolos a redoblar esfuerzos para tratar de hacer todo bien.

Los profesores formados en el campo de la ED sostuvieron que las principales causas de nerviosismo al inicio de su práctica ceñían la incertidumbre de no saber el tipo de profesores con quienes convivirían y la forma como serían recibidos, siendo evidente un trato indiferente hacia sus necesidades para controlar la disciplina o conocer estrategias de enseñanza al haber solicitado consejos a los profesores de mayor antigüedad y en su lugar era cuestionada su decisión de ingresar a la docencia en EMS o la eficacia de su desempeño, generando desolación e inseguridad conforme dicha situación se repetía. 
Finalmente, en promedio uno de cada diez profesores noveles por grupo comentó la preocupación que provocó haber sido llamado por el director previo a su ingreso al salón el mismo día que comenzaron a dar clases, por la autoridad que representa, a fin de conocerse, acompañarlos y presentarlos con los alumnos, siendo la única vez que mediaran palabras entre ellos. Fuera de estos casos, ninguno de los restantes nueve recibió algún tipo de acompańamiento desde que iniciaron su ejercicio docente.

\section{FACTORES DEL «CHOQUE CON LA REALIDAD»}

Derivado de las narrativas y las respuestas documentadas en cada entrevista focalizada, se logró identificar los principales Factores (positivo/negativo) que incidieron en la percepción del «choque con la realidad» por los profesores noveles al transitar la EIE y el ejercicio de la enseńanza profesional, así como sus elementos constitutivos que se correlacionan con los ámbitos de la práctica percibidos de mayor o menor conflicto cuando confrontaron las múltiples situaciones emergentes del contexto escolar y el intenso aprendizaje práctico obtenido, que se resumen en la tabla 7.

\begin{tabular}{|c|c|c|}
\hline \multicolumn{3}{|c|}{ TABLA 7. FACTORES DEL «CHOQUE CON LA REALIDAD» } \\
\hline FACTOR & Positivo & Negativo \\
\hline Materia a Enseñar & Conocida/Afín a su carrera profesional & $\begin{array}{l}\text { Desconocida/Ajena a su carrera } \\
\text { profesional }\end{array}$ \\
\hline Contenido & Práctica/sabiendo planificar & Teórica/sin dominar la planificación \\
\hline Horario & Primera Hora & Última Hora \\
\hline Turno & Matutino & Vespertino/Nocturno \\
\hline Semestre & $1 .^{\circ}-2 .^{\circ}$ & $5 .^{\circ}-6 .^{\circ}$ \\
\hline Género del grupo & Coincidente al profesor & Opuesto al profesor \\
\hline Edad del profesor & $\begin{array}{l}\text { Mayor juventud/Mayor Empatía/ } \\
\text { Menor Autoridad }\end{array}$ & $\begin{array}{l}\text { Menor Juventud/Menor empatía/ } \\
\text { Mayor Autoridad }\end{array}$ \\
\hline $\begin{array}{l}\text { Carácter del profesor y } \\
\text { aplicación de medidas } \\
\text { disciplinarias }\end{array}$ & $\begin{array}{l}\text { Marcar distancia con el grupo/ } \\
\text { Facilidad en el control de alumnos } \\
\text { problemáticos }\end{array}$ & $\begin{array}{l}\text { Preferir hacerse amigo de ellos/ } \\
\text { Dificultad en el control de alumnos } \\
\text { problemáticos }\end{array}$ \\
\hline $\begin{array}{l}\text { Amistad o parentesco } \\
\text { con personal de la escuela }\end{array}$ & $\begin{array}{l}\text { Presencia de profesores, jefes o } \\
\text { directivos que brinden consejos sobre } \\
\text { actividades escolares o administrativas }\end{array}$ & $\begin{array}{l}\text { Ausencia de profesores, jefes o directi- } \\
\text { vos que brinden consejos sobre activi- } \\
\text { dades escolares o administrativas }\end{array}$ \\
\hline Inicio con el ciclo escolar & $\begin{array}{l}\text { A la par de sus alumnos con el ciclo } \\
\text { escolar }\end{array}$ & $\begin{array}{l}\text { En fecha posterior al inicio del ciclo } \\
\text { escolar }\end{array}$ \\
\hline $\begin{array}{l}\text { Incorporarse como } \\
\text { reemplazo }\end{array}$ & $\begin{array}{l}\text { Siendo objeto de comparaciones y defi- } \\
\text { nido como «mejor, más preparado» }\end{array}$ & $\begin{array}{l}\text { Siendo objeto de comparaciones y defi- } \\
\text { nido como "peor, menos preparado" }\end{array}$ \\
\hline Proceso de Inserción & $\begin{array}{l}\text { Expectativa de recibir capacitación pre- } \\
\text { via mediante un programa de carácter } \\
\text { oficial antes del inicio de su práctica }\end{array}$ & $\begin{array}{l}\text { Aprendizaje individual sobre las } \\
\text { rutinas, trámites, procedimientos, } \\
\text { ambientación del centro escolar }\end{array}$ \\
\hline
\end{tabular}

Fuente: elaboración propia. 
Este factor representó el ámbito más problemático para la totalidad de los participantes, llegando a causar distintos niveles de ansiedad, nerviosismo e inquietud desde moderados hasta aprehensivos, aun cuando existiera un paralelismo entre su formación de origen y los contenidos a enseñar o por el contrario fueran disciplinas ajenas a sus perfiles de egreso, dado que en algunos casos eran asignadas materias que no siempre coincidían con su área del conocimiento, en todo caso emergían sentimientos de inseguridad al imaginarse frente a grupo sin dominar la materia o evidenciar una falta de preparación e inexperiencia ante sus alumnos al responder cuestionamientos de manera imprecisa.

\section{Contenido}

La dificultad centrada en el tratamiento de los contenidos fue resentida en mayor medida por los profesores noveles con formación ajena a la enseñanza, debido al desconocimiento de las etapas secuenciadas del proceso enseñanza-aprendizaje desde algunos elementos de forma como el diseño de una planificación según los ítems en los que se divide hasta otros de fondo mucho más complejos, acorde con los propósitos formativos de la materia, la organización de las actividades, los momentos didácticos de la clase, la dosificación de los tiempos, los productos de aprendizaje a obtener, los instrumentos de evaluación e inclusive si consideraba solo la transmisión de teoría sin la posibilidad de incluir actividades prácticas o por el contrario utilizaban materiales o equipo especial.

\section{Horario}

El lapso temporal que cubrían los profesores al atender sus materias estaba condicionado a una asignación indiscriminada que no regulaba programarlos en horas consecutivas o un solo turno, de ahí que el contraste entre los grupos de las primeras horas de clase los percibía accesibles y poco demandantes por sus alumnos con una sensación de que estaban "frescos» por haberse levantado temprano, en algunos casos trabajando todavía sin luz de día. A diferencia de la última clase, la cual fue descrita como la más difícil de cubrir debido al «fastidio» que reflejaban los alumnos sin el menor interés de seguir dentro de un salón para atender la clase después de una jornada completa, lo que derivaba en actitudes irreverentes bajo una única exigencia: «Salir de una vez por todas». 
Los múltiples subsistemas que integran la EMS permiten la distribución de sus actividades en diferentes turnos: a) Matutino, b) Vespertino o c) Nocturno; según la organización específica del centro escolar. Cada uno de ellos fue señalado por los participantes como generador de distintos niveles de estrés, en tanto, el trabajo docente transcurría sin menor complicación al trabajar por la mañana con los alumnos que no eran muy indisciplinados o irrespetuosos en cambio, los horarios vespertinos o nocturnos generaban una percepción de dificultad casi inaguantable ante comportamientos fuera de control.

\section{Semestre}

Los primeros dos semestres fueron identificados de dificultad moderada por los participantes que iniciaron con alumnos en este nivel escolar, al suponer que se encuentran descubriendo una nueva etapa de su vida, en una escuela con modalidades, compañeros y rutinas que desconocen, a diferencia de los últimos dos semestres (quinto y sexto), donde el ambiente del aula y la interacción con los alumnos lo hallaron más ríspidos más aún por encontrarse en la recta final de sus estudios conocen las prácticas de la escuela y manifiestan expresiones de menosprecio hacia las evaluaciones finales o los contenidos curriculares que no tienen aplicación en la vida real, ya que, en sus palabras, «a final de cuentas van a graduarse de todos modos». Mostrándose en algunos casos más interesados en la fiesta de graduación que en cumplir con las actividades escolares.

\section{GÉNERO DEL GRUPO OPUESTO AL PROFESOR(A)}

En algunos casos, atender una mayoría de hombres o mujeres integrantes del grupo bajo su responsabilidad les causó algún tipo de incomodidad a los profesores noveles al no estar acostumbrados a tratar con adolescentes y las características propias del género opuesto, en el caso específico de profesoras atendiendo grupos de ingeniería con mayoría de alumnos hombres los comportamientos exhibidos dentro de la jornada escolar diaria emergían mediante bromas de doble sentido, palabras groseras o subidas de tono e inclusive incitar pláticas de índole sexual sin el menor respeto hacia ellas, bajo este ambiente problemático la práctica docente se dificultaba debido a la molestia que les causaba, además de no estar habituadas a interactuar con una gran audiencia. 
Este factor en particular puede ser descrito desde dos perspectivas a partir de una relación Edad/Empatía/Autoridad, ya que la evidente juventud que tenían algunos profesores noveles permitía alcanzar un nivel de empatía con sus alumnos suficiente como para confiarles que estaban muy a gusto con ellos a pesar de reflejar inexperiencia y una falta de autoridad que motivaba falta de respeto; por otro lado, quienes tenían una edad mayor eran vistos como personas que si bien sabían mucho no los comprendían, reflejaban autoridad y mantenían el control de la disciplina con «mano dura».

\section{CARÁCTER DEL PROFESOR Y APLICACIÓN DE MEDIDAS DISCIPLINARIAS}

Los primeros contactos que tuvieron los profesores noveles con el contexto escolar y la cultura propia de la institución estuvieron enmarcados por el desarrollo de un sentimiento exponencial de nerviosismo desde antes de comenzar sus clases que se agudizaba una vez habiendo ingresado al aula, ya que significaba confrontar la relación docente/discente en sus inicios con el apremio de ganarse su confianza sin utilizar medidas disciplinarias estrictas para alcanzar un ambiente cordial, no ser vistos como dictadores escolares y facilitar su enseñanza; no obstante, irrumpía un dejo de desilusión cuando los resultados no eran los esperados y en su lugar observaban que el salón se convertía en "tierra de nadie» a tal punto que el seguimiento de sus actividades les parecía casi imposible.

\section{Amistad o parentesco Con personal de la ESCUEla}

Se documentó la existencia de un marcado sentimiento de soledad en la mayoría de los profesores noveles, debido a la ausencia de algún familiar o conocido que trabajara en el mismo centro escolar para apoyarlos en el conocimiento de las rutinas institucionales básicas, les compartiera algunas estrategias de enseñanza, medidas de control disciplinar, trámites administrativos o por lo menos ofreciera un soporte emocional; en contraparte, la presencia de alguna persona vinculada familiar o socialmente era vista como una ventaja práctica en su tránsito inicial, considerando que además de auxiliarlos cubrían la inexistencia de un programa institucional que facilitara su inserción.

\section{INICIO CON EL CICLO ESCOLAR}

La recurrente eventualidad de no haber comenzado el ejercicio de la enseñanza por algunos profesores noveles con el ciclo escolar a la par de sus alumnos generó múltiples desasosiegos al verse rebasados temporalmente en la cobertura de los contenidos, más aún cuando el centro escolar tenía la recurrente omisión de faci- 
litar los programas de sus materias y padecían la dificultad de planear con la premura de darles continuidad a sus actividades, en ocasiones apoyadas en el trabajo de un semestre anterior, además de aplicar las evaluaciones parciales en tiempo y forma conforme lo marcaba el calendario institucional; en contraparte, si comenzaban su ejercicio a la par del ciclo escolar disminuían las anteriores preocupaciones con la ventaja de que podían «aprender todos juntos».

\section{INCORPORARSE COMO REEMPLAZO}

En algunos casos, la inserción profesional a la enseñanza no ocurría para cubrir una plaza o un interinato sino que se ingresaba como reemplazo del profesor titular ante su ausencia por diversos motivos, de ahí que la generación de un ambiente propicio para la enseñanza se veía mediado positiva o negativamente por los constantes señalamientos de los alumnos en torno a las comparaciones que hacían con el profesor anterior, es decir, durante la interacción con los alumnos emergían puntuales señalamientos sobre su desempeño, dominio de la materia, facilidad de palabra e inclusive apariencia física, todo esto bajo la «medición» efectuada bajo una calificación comparativa entre ambos profesores, definida según el nivel de superioridad o inferioridad expuesto.

\section{Proceso de inserción}

Se documentó en la totalidad de los participantes una creencia firme de que existiría un programa de inducción o acompañamiento previo al inicio de su práctica docente para habilitarlos pedagógica, administrativa e institucionalmente, por parte de cada centro escolar; sin embargo, una vez incorporados en sus actividades se dieron cuenta de lo equivocada que estaba dicha suposición al verse en solitario mientras se incrementaban sus dudas y preocupaciones sobre el ejercicio de la enseñanza o los trámites administrativos, de ahí que la integración progresiva en la cultura escolar constituyó una impronta de mucho valor formativo con la que afirmaron haberse «hecho profesores» ellos solos.

\section{MODELO DE CHOQUE DIVERGENTE}

Los factores positivos/negativos que fueron identificados por tener una influencia determinante en la percepción del «choque con la realidad» de los profesores noveles de EMS al transitar la EIE fueron objeto de un proceso de ruptura y transformación a partir del análisis cíclico aislamiento/relación entre sus componentes, apartándolos de su ambiente caótico de origen para elaborar un diseño abstracto que exteriorizara el orden subyacente mediante un modelo teórico integrado con las propiedades del fenómeno estudiado, exponiendo la interrelación existente entre ellos, según la intensidad socioemocional que genera confrontar las desavenen- 


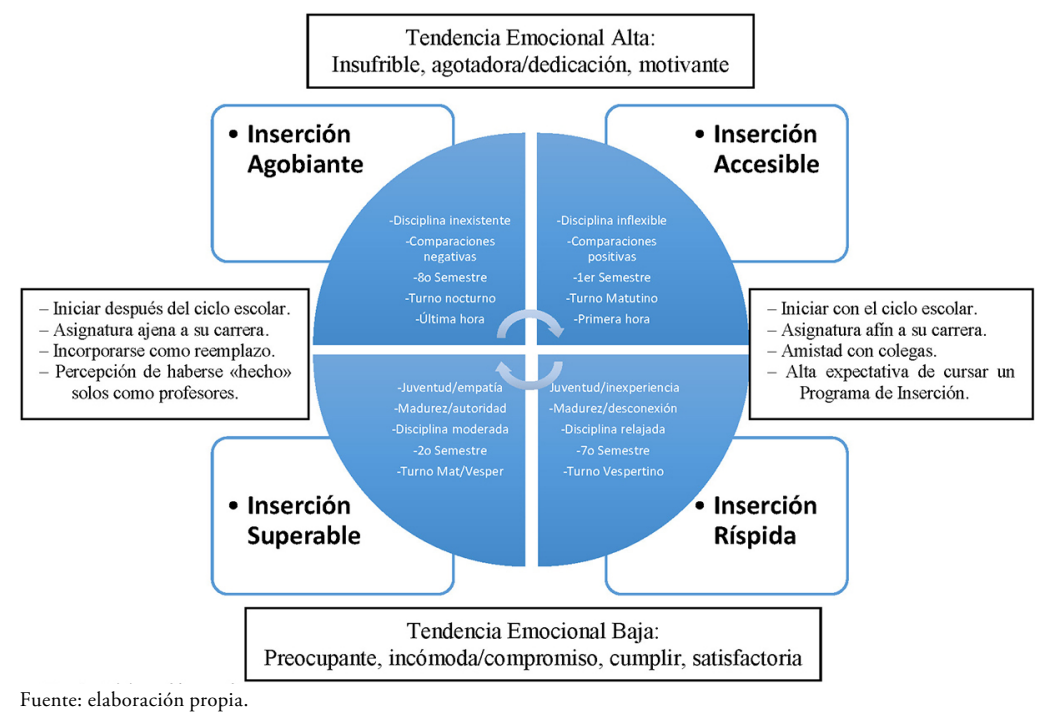

Figura 1. Modelo de Choque Divergente.

cias de la enseñanza profesional; en tanto, los factores que minimizan o maximizan su efecto divergen de las características personales y profesionales del profesor, la cultura del centro escolar, las relaciones interpersonales, a la vez que definen el nivel de «aspereza» que tendrá su proceso de inserción; lo cual se muestra en la figura 1 .

El modelo presentado en la figura 1 da cuenta de un movimiento constante de procesos, fenómenos y circunstancias bajo distintos niveles de vinculación, subordinación o cooperación, de ahí que las mediaciones observables entre la Tendencia Emocional Alta o Baja se correlacionan con la organización interna de sus factores y elementos a partir de un automovimiento que denota las situaciones clave definitorias del tamiz que podrán adquirir los cuatro tipos de Inserción integrantes del modelo (Agobiante, Ríspida, Accesible, Superable) según la divergencia de sus nexos con el contexto escolar.

En la parte alta se encuentra la interacción entre factores que denotan una inserción insufrible o agotadora, es decir, diversos elementos de la práctica docente que la hacen sumamente desgastante y por momentos tan inaccesible que se llega a desear abandonarla, por otro lado están los que permiten descubrirla accesible, sin mayores problemas o conflictos, generando suficiente motivación para dedicarle esfuerzo. La parte baja ubica elementos con interacciones ríspidas, donde se encuentra un escenario que en principio parece inaccesible, preocupante o incómodo pero con el paso del tiempo y el cúmulo de experiencia promueve adaptarse a las condiciones de trabajo; por último, están los superables, entre lo que destaca asumir un compromiso para cumplir la enseñanza profesional sin necesitar mayor apremio que la propia capacidad de adaptación al entorno escolar.

Si bien el modelo presentando trata de integrar todos los ámbitos, factores y elementos documentados, se debe tener en cuenta que la dificultad de conocer 
por completo un fenómeno social ante su naturaleza cambiante y contextualizada requiere aplicar «incisiones» a sus particularidades; por ello, se puede señalar que su diseño toma en cuenta las características dinámicas que son propias a la enseñanza profesional, a fin de que no resultara un bosquejo estático o impreciso, por el contrario, si bien representa el "clímax» de esta investigación pretende servir de punto de partida para posteriores investigaciones que lo recuperen como referente en el estudio del profesor novel, el «choque con la realidad», la EIE, etc., al advertir factores, elementos o relaciones emergentes.

\section{CONCLUSIÓN}

Las eventualidades que rodean la etapa de inserción profesional en EMS, a la luz de los resultados obtenidos, nos permiten confirmar la relevancia que tiene este periodo para el profesor novel por ser el marco de un proceso indefinido tanto en su atención institucional como relevante desde el aprendizaje individual, condicionado por un contexto donde recibe de súbito toda la responsabilidad de un grupo al considerarlo profesor en toda la extensión de la palabra, lo cual contrastaría si supone un segundo proceso de inserción, es decir, posterior a uno preliminar habiendo iniciado progresivamente el ejercicio de su profesión de origen con actividades sencillas hasta dominar otras más complejas y los "gajes del oficio».

Por ello, los problemas confrontados en una cultura escolar con escenarios, rutinas, interacción y exigencias conocidas hasta cierto punto como estudiante pero desconocidas como profesor titular, son reflexionadas por el profesor novel a partir de los saberes construidos en las etapas formativas previas según vaya recuperando imágenes significativas de la práctica docente que suponga transferibles para resolver las contingencias de la enseñanza en la EMS. En este sentido, dicha cobertura de vacíos didácticos o disciplinares pone de manifiesto la necesaria articulación que demanda el periodo de inserción con las etapas anteriores por ser un momento coyuntural donde el incipiente aprendizaje de la enseñanza definirá una identidad docente específica.

Más aún cuando la doble actividad propia de la inserción, Enseñar y Aprender a Enseñar, no se reduce al ejercicio del proceso enseñanza-aprendizaje o los intensos contactos entre profesor y alumnos en el aula, sino que se extiende hacia un contexto mucho más amplio que requiere la apropiación de rasgos socioculturales correspondientes a la comunidad donde se encuentre el centro escolar, así como sus normas, usos y costumbres del subsistema que forme parte en la EMS, que establecen distintos niveles de interacción con autoridades educativas, el personal administrativo y los compañeros profesores como parte del «ritual» que conlleva iniciar las relaciones laborales desde su llegada, convergiendo rasgos de índole personal cuando la lejanía requiera alejarse de su hogar, la familia, los amigos, pareja, entre otros.

Así pues, la inserción del profesor novel está articulada entre ámbitos a) personales: donde la interacción que efectúa con sus alumnos se define como resultado de una relación edad-empatía-autoridad; b) curriculares: relacionando la naturaleza de la materia y la complejidad del contenido con el desconocimiento del pro- 
ceso enseñanza-aprendizaje; c) áulicos: producto del ambiente que resulta de alumnos con mayoría de género opuesto, utilizar medidas disciplinarias flexibles/rígidas, además de cuestionarlo si reemplazó a otro profesor; y d) institucionales: a partir de la asignación del turno, horario, semestre sin considerar su poca o nula experiencia, si existió algún trabajador del centro escolar que le acompañara y la ausencia de un programa oficial de inducción; en resumen, cada uno da cuenta de la intensa y contradictoria transición que representa el inicio del aprendizaje de la enseñanza profesional cuando se confronta el «choque con la realidad».

Recibido: 17-04-2019. Aceptado: 24-05-2019 


\section{REFERENCIAS BIBLIOGRÁFICAS}

Aguirre, S. (1995). «Entrevistas y cuestionarios», en Aguirre, A. (ed.). Etnografía. Metodología cualitativa en la investigación sociocultural. Barcelona: Alfaomega.

Berliner, D. (1984). «The Half-Full Glass: A Review of Research on Teaching», en Hosford, P. (ed.), Using What We Know About Teaching (pp. 51-81). Virginia: Association for Supervision and Curriculum Development.

Воотн, T. (1996). «Sounds of still voices: Issues in the use of narrative methods with people who have learning difficulties», en Barton, L. (ed.). Disability and Society. Emerging Issues and Insights (pp. 237-255). New York: Logman Publishing.

Bolívar, A. y otros (1998). La investigación biográfico-narrativa en educación. Guia para indagar en el campo. Granada: FORCE.

Bullough, R. (2000). «Convertirse en profesor: la persona y la localización social de la formación del profesorado", en B. Biddle, B., Good, T. y Goodson, I. (eds.). La enseñanza y los profesores I (La profesión de enseñar) (pp. 99-166). Barcelona: Paidós.

Clark, C. y Yinger, R. (1979). Three studies of teacher planning. East Lansing: Institute for Research on Teaching, Michigan State University.

Clark, C. y Yinger, R. (1980). The hidden world of teaching: Implication of research on teacher planning. East Lansing: Institute for Research on Teaching, Michigan State University.

Collins, B. y Winnip, K. (2002). «Two scenarios for productive learning environments in the workplace». British Journal of Educational Technology, 33(2), 133-148.

Connelly, F.M. y Clandinin, D.J. (1995). «Relatos de experiencias e investigación narrativa», en Larrosa, J. y otros (eds.), Déjame que te cuente. Ensayos sobre narrativa y educación. Barcelona: Laertes.

DAY, C. (2005). Formar Docentes: cómo, cuándo y en qué condiciones aprende el profesorado. Madrid: Narcea.

Diario Oficial de la Federación (2013). «Decreto por el que se expide la Ley General del Servicio Profesional Docente», tomo DCCXX, n. ${ }^{\circ} 8$, segunda sección, título segundo, capítulo I, art. 22, párrafo segundo.

Eirín, N., Montero, M. y García, R. (junio de 2008). Profesores Principiante e Iniciación Profesional. Estudio Exploratorio. Trabajo presentado en I Congreso Internacional sobre Profesorado Principiante e Inserción Profesional a la Docencia. Sevilla, España: Universidad Autónoma de Sevilla, Facultad de Ciencias de la Educación.

Esteve, J.M. (1997). La formación inicial de los profesores de secundaria. Barcelona: Ariel.

Feiman-Nemser, S. (2001). «Helping novices learn to teach». Journal of Teacher Education, vol. 52, (1), $17-30$.

Festinger, L. (1957). A Theory of Cognitive Dissonance. Evanston IL: Row, Peterson \& Company.

Gilroy, P. (1993). «El Conocimiento Profesional y el Profesor Principiante», en CARr, W. (ed.). Calidad en la Enseñanza e Investigación Acción (pp. 87-100). Sevilla: Díada Editorial.

Huberman, M., Thompson, C.L. y Weiland, S. (2000). «Perspectivas de la carrera del profesor», en Biddle, B., Good, T. y Goodson, I. (eds.). La enseñanza y los profesores. La profesión de enseñar (pp. 19-879). Barcelona: Paidós. 
Hunter, M. (1984). «Knowing, Teaching and Supervising», en Hosford, P. (ed.). Using What We Know About Teaching (pp. 169-192). Virginia: Association for Supervision and Curriculum Development.

Imbernón, F. (2007). La Investigación Educativa como herramienta de formación del Profesorado. Reflexiones y experiencias de investigación educativa. Barcelona: Grao.

Marcelo, C. y Mingorance, P. (1992). Pensamiento de profesores y desarrollo profesional II. Formación inicial y permanente. Universidad de Sevilla: Instituto de Ciencias de la Educación, n. ${ }^{\circ} 8$.

Marcelo, C. (1987). El pensamiento del profesor. Barcelona: Editorial CEAC.

Marcelo, C. (1999). «Estudios sobre Estrategias de Inserción profesional en Europa». Revista Iberoamericana de Educación, Organización de Estados Iberoamericanos, (19), 101-144.

Marcelo, C. (2007). «Empezar con buen pie: inserción a la enseñanza para profesores principiantes». Revista Docencia, 33, XII.

Marcelo, C. (2008). El Profesorado Principiante: Inserción a la docencia. Barcelona: Octaedro.

Minichiello, V., Aroni, R., Timewell, E. y Alexander, L. (1990). In-depth Interwing. Melbourne: Logman.

Newman, I. y Benz, C. (1998). Qualitative-Quantitative Research Methodology. Exploring the Interactive Continuum. Carbondale \& Edwardsville: Southern Illinois University Press.

Tashakrori, A. y Teddlie, C. (1998). Mixed Methodology. Combining Qualitative and Quantitative Approaches. London: SAGE Publications, Applied Social Research Methods Series, vol. 46.

UNAM (2009). «Planes de estudio, Áreas de Conocimiento, Ciclo 2009-2010», disponible en http:// www.dgae.unam.mx/planes/carreras.html.

Veenman, S. (1984). «Perceived Problems of Beginning Teachers». Review of Education Research, 54 (2), 143-178.

Vonk, J.H.C. (1983). «Problems of the Beginning Teachers». European Journal of Teacher Education, 6 (2), 133-150.

Vonk, J. (1996). «A Knowledge Base for Mentors of Beginning Teachers: Results of a Dutch Experience», en McBridge, R. (ed.). Teacher Education Policy (pp. 112-134). London: Falmer Press. 